\title{
乌ூ \\ Atomic-scale magnetism of cobalt-intercalated graphene
}

\author{
Régis Decker, ${ }^{1}$ Jens Brede, ${ }^{1,{ }^{*}}$ Nicolae Atodiresei, ${ }^{2, \dagger}$ Vasile Caciuc, ${ }^{2}$ Stefan Blügel, ${ }^{2}$ and Roland Wiesendanger ${ }^{1}$ \\ ${ }^{1}$ Institute of Applied Physics, University of Hamburg, Jungiusstrasse 11, D-20355 Hamburg, Germany \\ ${ }^{2}$ Peter Grünberg Institut and Institute for Advanced Simulation, Forschungzentrum Jülich, D-52425 Jülich, Germany
}

(Received 4 July 2012; published 7 January 2013)

\begin{abstract}
Using spin-polarized scanning tunneling microscopy and density functional theory, we have studied the structural and magnetic properties of cobalt-intercalated graphene on $\operatorname{Ir}(111)$. The cobalt forms monolayer islands being pseudomorphic with the $\operatorname{Ir}(111)$ beneath the graphene. The strong bonding between graphene and cobalt leads to a high corrugation within the Moire pattern which arises due to the lattice mismatch between the graphene and the Co on $\operatorname{Ir}(111)$. The intercalation regions exhibit an out-of-plane easy axis with an extremely high switching field, which surpasses the significant values reported for uncovered cobalt islands on $\operatorname{Ir}(111)$. Within the Moiré unit cell of the intercalation regions, we observe a site-dependent variation of the local effective spin polarization. State-of-the-art first-principles calculations show that the origin of this variation is a site-dependent magnetization of the graphene: At top sites the graphene is coupled ferromagnetically to the cobalt underneath, while it is antiferromagnetically coupled at fcc and hcp sites.
\end{abstract}

DOI: 10.1103/PhysRevB.87.041403

PACS number(s): 73.22.Pr, 68.37.Ef, 75.75.-c

The electronic properties of graphene ${ }^{1}$ depend critically on its environment and in particular on its substrate..$^{2-4}$ Therefore, it is important to be able to precisely design and control the physical properties of the graphene-substrate interfaces. Inspired by the extensively studied graphite intercalation compounds, ${ }^{5-8}$ an emerging method to engineer technologically relevant hybrid organic-metal interfaces is to intercalate specific elements at the interface. ${ }^{9,10}$ So far, most efforts in that direction have been employed to use intercalants to reduce the graphene-substrate interaction in order to keep the unprecedented exotic properties of bare graphene intact, ${ }^{11-13}$ open a gap at the Dirac point, ${ }^{14}$ or induce superconductivity. ${ }^{15,16}$ However, this approach has not yet been exploited to create an interstitial ferromagnetic layer and study its effects on the properties of graphene.

Being made of light atoms, the spin-orbit coupling in graphene is known to be very weak and the intrinsic magnetic properties of graphene are difficult to observe experimentally. Therefore, most studies concerning the magnetic properties of graphene have been focusing on its edges, defects, and impurities. ${ }^{17}$ Experimentally, however, many features predicted by theory were not observed yet and the magnetic properties of graphene in general remain an almost unexplored and challenging research topic. ${ }^{18,19}$ Since graphene is only one layer thick and is known to be an inert material, graphenebased ferromagnetic heterostructures are an ideal candidate for a new class of tunnel magnetoresistance (TMR $)^{20}$ or giant magnetoresistance $(\mathrm{GMR})^{21,22}$ devices. A detailed state-ofthe-art experimental and theoretical description at the atomic scale of the topography as well as of the complex phenomena occurring at the hybrid graphene-ferromagnetic surface and interface represents the first necessary steps towards the development of graphene-based spintronics.

In this combined experimental and theoretical study, we gain a unique and detailed insight into the physical properties of the cobalt-intercalated graphene/ $\operatorname{Ir}(111)$ system. Using spinpolarized scanning tunneling microscopy (SP-STM), ${ }^{23}$ we resolve simultaneously the morphology and the local magnetic properties of graphene on top of monolayer cobalt islands which were intercalated between the graphene and the $\operatorname{Ir}(111)$ substrate. State-of-the-art first-principles calculations were performed to gain a thorough insight into the nature of the local bonding and the magnetic interactions present at the hybrid graphene-ferromagnet interface that lead to the experimentally observed topography and local spin polarization in this system.

SP-STM measurements were performed under UHV conditions $\left(P_{\text {base }}<1.10^{-10} \mathrm{mbar}\right)$. All tip and sample preparations were done in vacuo. The $\operatorname{Ir}(111)$ substrate was prepared by repeated cycles of sputtering and annealing in $\mathrm{O}_{2}$ atmosphere $\left(P_{\mathrm{O}_{2}}=10^{-7} \mathrm{mbar}\right.$ ) followed by a flash at $\sim 1000^{\circ} \mathrm{C}$. After the cobalt intercalation process (see below), samples were transferred in situ into a home-built SP-STM operated at $\sim 6 \mathrm{~K}$. Fe-coated ( $\sim 50 \mathrm{ML})$ tungsten tips were used to observe the magnetic structure of the sample. All data were acquired in the constant-current mode. Spin-polarized differential tunneling conductance maps were recorded using the lock-in technique, by detecting the ac tunneling current induced by a sinusoidal voltage added to the dc sample bias $V_{b}$, with the magnetic orientation of the tip aligned by applying an external magnetic field $|B| \geqslant 1 \mathrm{~T}$. Calculations were performed via DFT in the generalized gradient approximation + Hubbard term $(\mathrm{GGA}+U)$, including van der Waals interactions..$^{24,25}$ As observed experimentally, ${ }^{26}$ the spin polarization above the $\mathrm{Co} / \mathrm{Ir}(111)$ surface and the out-of-plane magnetization direction is reproduced when using a $U=4 \mathrm{eV}$ in our calculations. Thus, this value has been chosen also for the graphene/Co/Ir(111) system. The graphene/Co/Ir(111) system was modeled by a $(10 \times 10)$ graphene unit cell $(200 \mathrm{C}$ atoms $)$ on a $(9 \times 9) \mathrm{Co} / \operatorname{Ir}(111)$ unit cell (one layer of $\mathrm{Co}$ and three layers of Ir, i.e., 81 Co atoms and 243 Ir atoms).

The $\operatorname{Ir}(111)$ surface was partially covered by micron-sized graphene patches obtained by a chemical vapor deposition as described in Ref. 27. On the one hand, graphene on transition metal systems such as $\mathrm{Ru}(0001)^{28,29}$ is dominated by strong chemical interaction between the graphene and the surface. On the other hand, the bonding of graphene on $\operatorname{Ir}(111)$ is primarily of van der Waals type, with a chemical modulation. ${ }^{30}$ A Moiré pattern arises in the STM topography due to the lattice 
mismatch between the graphene and the $\operatorname{Ir}(111)$ surface. ${ }^{31}$ In the absence of a twisting angle between the two lattices, the Moiré pattern exhibits a lattice parameter of $\sim 25 \AA$, as also observed in our experiments.

Cobalt was deposited onto the graphene/Ir(111) sample, which was kept at or slightly above room temperature. The intercalation occurs by annealing the graphene $+\mathrm{Co}$ covered $\operatorname{Ir}(111)$ surface at $\sim 200^{\circ} \mathrm{C}$ for several minutes. At the graphene-covered areas, this results in the formation of random sized but well-defined intercalation regions, mostly located at $\operatorname{Ir}(111)$ step edges. These intercalation regions are also characterized by a Moiré pattern with a high corrugation $(\sim 1.2-1.8 \AA$, depending on the tunneling
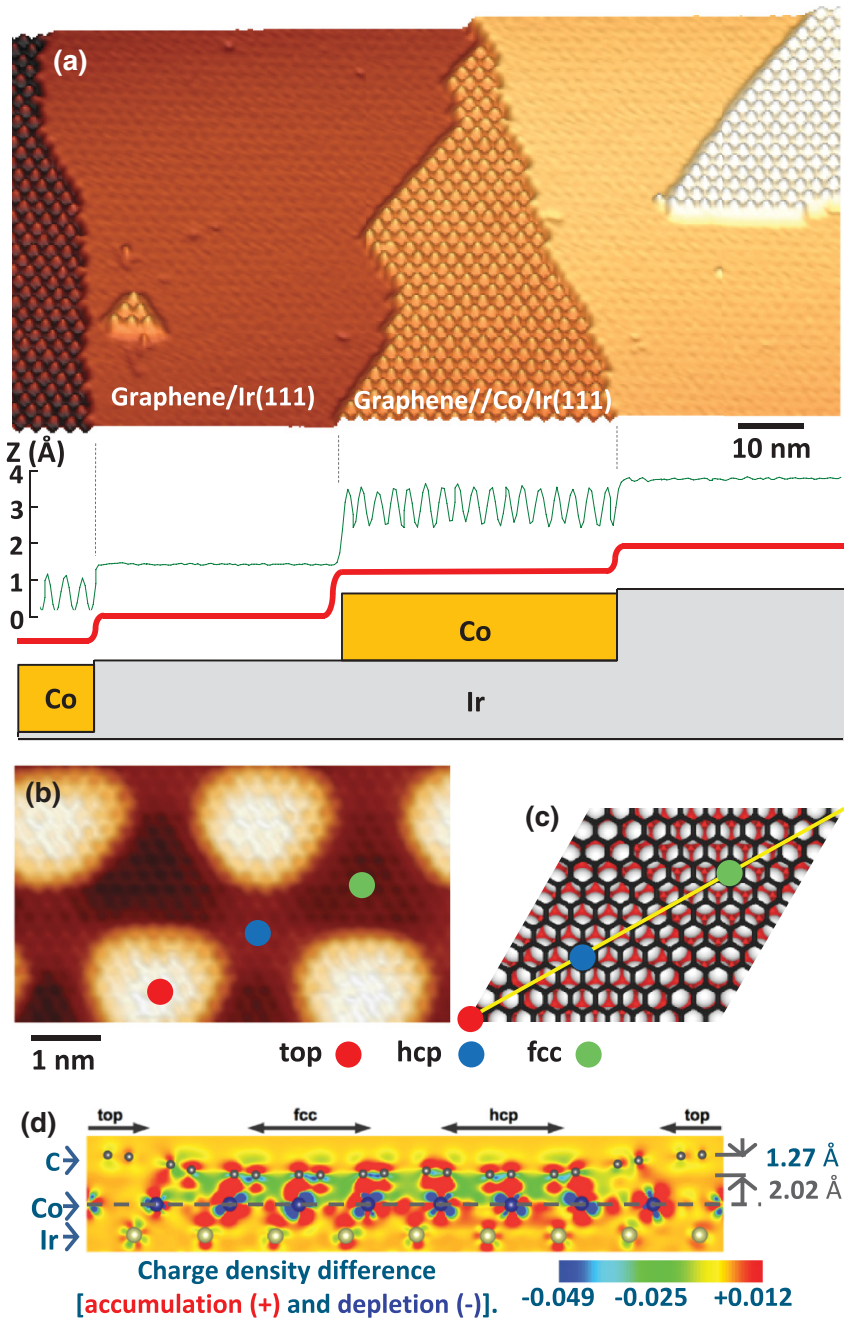

FIG. 1. (Color) Structure of cobalt-intercalated graphene on $\operatorname{Ir}(111)$. (a) Topography of the graphene/Ir(111) surface with four intercalation regions at typical locations $(-0.7 \mathrm{~V}, 1 \mathrm{nA})$. The line profile taken at the bottom of the topograph illustrates the difference between the graphene $/ \operatorname{Ir}(111)$ and the graphene/Co/Ir(111) Moiré corrugations. (b) High-resolution STM topograph $(-0.1 \mathrm{~V}, 4 \mu \mathrm{A})$ with the graphene atomic lattice and the Moiré pattern at a cobalt intercalation region. (c) Model of graphene/Co/Ir(111) (see text) showing the unit cell. Black: graphene, white: cobalt, red: iridium atoms. (d) Side view of the charge density difference plots of the graphene on $\mathrm{Co} / \operatorname{Ir}(111)$ surface cut along the yellow plane in (c) (colorbar units in $\mathrm{e} / \AA^{3}$ ). current and/or the bias voltage). A typical STM image of the graphene/ $\operatorname{Ir}(111)$ surface with intercalated cobalt areas is presented in Fig. 1(a). The line profile (green line) shown underneath Fig. 1(a) clearly illustrates the striking difference of the graphene/Ir(111) and the graphene/Co/Ir(111) Moiré corrugations. A magnified view from a high-resolution STM topograph in Fig. 1(b) highlights the morphology at the atomic scale of the graphene/Co/Ir(111) heterostructure [see also the ball-and-stick model in Fig. 1(c)]. The lateral periodicity and orientation of this Moiré pattern is identical to the one of graphene/Ir(111), which suggests that the cobalt layer underneath graphene is pseudomorphic with the $\operatorname{Ir}(111)$ lattice. From the ground-state geometry of the graphene/Co/Ir(111) system obtained by means of density functional theory (DFT), we find that the highest and lowest $\mathrm{C}$ atoms above the Co layer are $3.29 \AA$ at the top position and $2.02 \AA$ at the hcp sites, respectively. Therefore, the theoretical atomic (geometrical) graphene corrugation is $1.27 \AA$ [Fig. 1(d)] which is significantly larger than $0.41 \AA$ obtained for graphene/Ir(111). ${ }^{30,32}$ Note that in our STM experiments the measured corrugation is in the range of 1.2-1.8 $\AA$ and comparable with the geometrical one. ${ }^{33}$ Therefore, the measured corrugation mainly reflects the corrugation within the Moiré pattern of the graphene layer itself on top of the atomically flat intercalated cobalt layer.

During our experiments we noted that the cobalt intercalation areas seem to be located preferentially at small graphene patches or close to the border of bigger patches. In addition, the fact that intercalation areas are almost exclusively located at $\operatorname{Ir}(111)$ step edges [Fig. 1(a))] indicates a low migration barrier for cobalt at the interface. Although an intercalation mechanism, which would involve a penetration of Co atoms at graphene defects, cannot be ruled out, these observations suggest that in our experiments the formation of intercalated islands is due to the migration of cobalt atoms from the graphene patch edges. ${ }^{34}$

Detailed insights into the magnetic properties of the hybrid graphene/Co/Ir(111) system are revealed by our SP-STM experiments. Figure 2 presents two maps of the spin-resolved differential tunneling conductance ( $d I / d V$ map) of the same area with an out-of-plane applied magnetic field of $+1 \mathrm{~T}$ and $-1 \mathrm{~T}$, respectively. These magnetic fields are strong enough to align the magnetization direction of the Fe-coated tip but are too small to align the magnetization direction

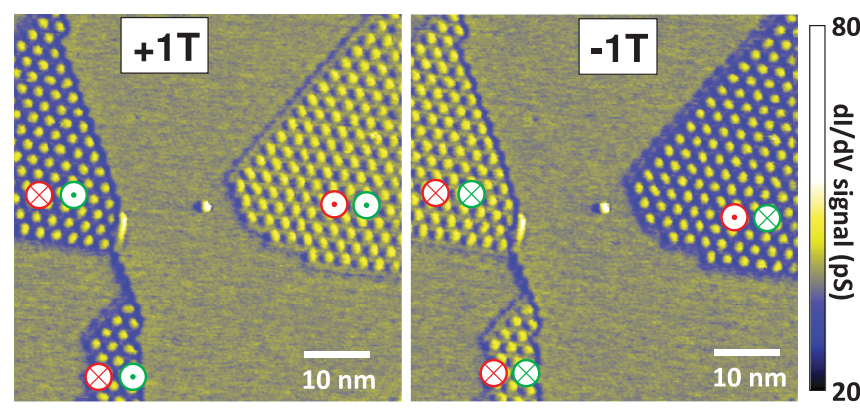

FIG. 2. (Color) Out-of-plane magnetic maps of the intercalation regions. Spin-resolved $d I / d V$ maps $(-0.9 \mathrm{~V}, 1 \mathrm{nA})$ acquired with an applied out-of-plane magnetic field. Green symbols: magnetic orientation of the SP-STM tip, red symbols: magnetic orientation of the intercalation regions. 
of the graphene/Co/Ir(111) heterostructures (see for example Ref. 26). For opposite field orientations we observe a contrast reversal for the intercalation regions in the spin-resolved $d I / d V$ maps. The contrast reversal arises due to the tunneling magnetoresistance (TMR) effect ${ }^{20}$ and unambiguously establishes the spin sensitivity in the measurements. More precisely, the contrast is due to a difference between the majority and minority density of states (DOS) at this particular energy measured at the position of the tip. ${ }^{35}$ Therefore, primarily, the electronic states that have a long range decay into the vacuum give rise to the observed contrast. No spin contrast has been observed while applying an in-plane magnetic field, which clearly indicates a magnetic anisotropy of the heterostructure with an out-of-plane easy axis.

To characterize the response of the system to an external magnetic field and to get an estimate of the magnetic anisotropy energy, we performed a series of spin-polarized $d I / d V$ measurements of large surface areas containing 33 intercalation regions of different sizes from $\sim 40 \mathrm{~nm}^{2}$ to $\sim 2930 \mathrm{~nm}^{2}$ and various shapes. Each subsequent map was acquired with a different out-of-plane magnetic field in the following order: $0 \mathrm{~T} \rightarrow-6.5 \mathrm{~T} \rightarrow+6.5 \mathrm{~T} \rightarrow-6.5 \mathrm{~T}$. The percentage of area among all intercalation regions which are aligned in the $+z$ direction vs the applied field is plotted in Fig. 3 and shows a hysteresis loop. It indicates that even for an applied field as high as $\pm 6.5 \mathrm{~T}$ (our upper field limit), we are not able to align $100 \%$ of the intercalation regions. Therefore, the actual coercive field is in reality higher than the value of $\sim 4.5 \mathrm{~T}$ deduced from the loop and much higher than the one measured for $\mathrm{Co} / \operatorname{Ir}(111){ }^{26}$ This clearly shows that the graphene/Co/Ir(111) heterostructures have a much higher magnetic anisotropy energy than cobalt islands on $\operatorname{Ir}(111)$.

Experimentally, we observe an out-of-plane magnetic anisotropy for the $\mathrm{Co} / \operatorname{Ir}(111)^{26}$ and the graphene/Co/Ir(111) systems. The out-of-plane magnetic anisotropy is also well known from previous reports concerning molecular adsorbates on ferromagnetic layers. ${ }^{37}$ More importantly, we also observe an enhancement of this physical quantity for the graphene/Co/Ir(111) system compared to $\mathrm{Co} / \mathrm{Ir}(111)$ (see

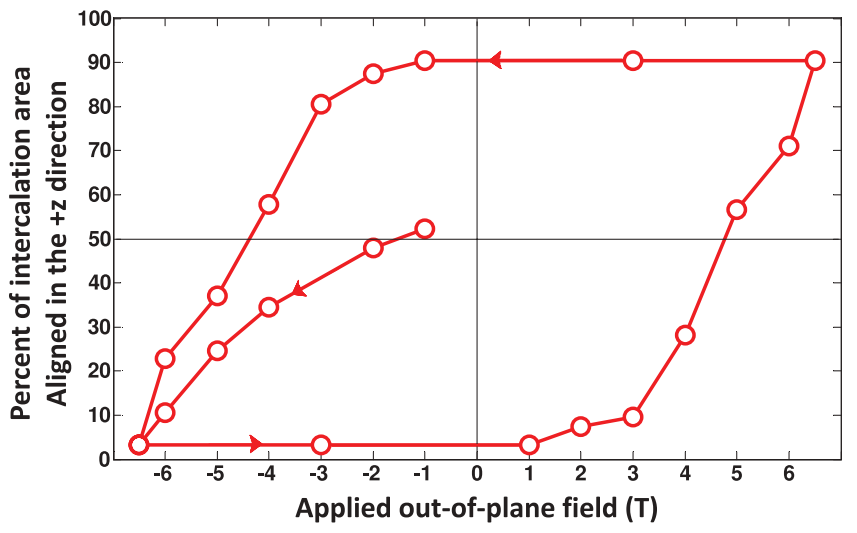

FIG. 3. (Color) Coercivity of intercalation regions. Hysteresis loop obtained from large-scale spin-resolved $d I / d V$ maps presenting 33 intercalation regions (Ref. 36). Each point represents the intercalation area, in percent, among the one, which is aligned with the magnetic field, for a sequence of different magnetic fields (see arrows).
Fig. 3 and Ref. 26). This enhancement originates from the fact that this system presents strong interface effects such as charge transfer that induces a net magnetization in the adsorbed graphene. Although from the computational point of view the evaluation of magnetic anisotropy for the graphene/Co/Ir(111) is still a challenge, a similar effect was demonstrated in one of our previous studies where an organic molecule becomes magnetic after its adsorption onto a ferromagnetic surface and enhances the out-of-plane magnetic anisotropy of the entire system. $^{38}$

In the following, based on the theoretical simulations we will discuss the origin of the atomic and magnetic structure observed in our SP-STM experiments. The calculated C-Co interatomic distances of $\sim 2.0 \AA$ and the charge density difference plot at fcc and hcp sites indicate a strong cobaltcarbon hybridization and the formation of a chemical bond. Note that by comparing the charge density difference plots of the graphene on $\operatorname{Ir}(111)^{30,36}$ and on $\mathrm{Co} / \operatorname{Ir}(111)$ surfaces [Fig. 1(d)] the hybridization between cobalt and carbon atoms at these sites is much stronger than the carbon-iridium one. The calculated C-Co interatomic distances of $\sim 3.5 \AA$ at top sites corresponds to a much weaker interaction dominated by van der Waals bonding [see Fig. 1(d) and Ref. 36. For the relaxed geometry, the DFT binding energy contribution is repulsive (+5.0 meV per $\mathrm{C}$ atom), while the van der Waals binding energy is attractive $(-107.0 \mathrm{meV}$ per $\mathrm{C}$ atom). Therefore, the total calculated binding energy is $-102.0 \mathrm{meV}$ per carbon atom which is almost twice as large as for graphene/ $\operatorname{Ir}(111){ }^{30}$

We address now the question of the origin of the observed pattern of the local spin polarization of the heterostructures. The magnetic ground state of the cobalt layer beneath the graphene is calculated to be ferromagnetic with an out-ofplane easy axis and with a magnetic moment of the cobalt atoms at the hcp, fcc, and top sites of $\sim+1.92 \mu_{B}\left(\mathrm{Co}^{\mathrm{hcp}}\right)$, $\sim+1.88 \mu_{B}\left(\mathrm{Co}^{\mathrm{fcc}}\right)$, and $\sim+2.08 \mu_{B}\left(\mathrm{Co}^{\text {top }}\right)$ due to a larger number of electrons in the spin-up channel than in the spindown one. However, as can be seen in the maps of Fig. 2 acquired at $V_{b}=-0.9 \mathrm{~V}$ the top sites and fcc/hcp sites of the graphene/Co/Ir(111) Moiré pattern unit cell present a different spin-resolved signal of the differential tunneling conductance, i.e., a difference in the majority and minority density of states at the tip position for this energy. To get a deeper insight into this feature, we acquired high-resolution spin asymmetry maps

$$
A(e V, r)=\frac{\left[\frac{d I}{d V}(e V, r)\right]_{\uparrow \uparrow}-\left[\frac{d I}{d V}(e V, r)\right]_{\uparrow \downarrow}}{\left[\frac{d I}{d V}(e V, r)\right]_{\uparrow \uparrow}+\left[\frac{d I}{d V}(e V, r)\right]_{\uparrow \downarrow}}
$$

of an intercalation region, comparable to those shown in Figs. 1, 2, and 3 ( $e$ is the elementary charge, $V$ the sample bias, $I$ the tunnel current, and the $\uparrow \uparrow$ and $\uparrow \downarrow$ symbols denote a parallel and antiparallel alignment, respectively, of the magnetization directions of the tip and the intercalation region). Such a map obtained for an energy of $-1.0 \mathrm{eV}$ is shown in Fig. 4(b) and reveals an inversion of the local effective spin polarization between the top sites and the fcc/hcp sites which defines a magnetic Moiré pattern for this bias voltage. The simulated surface spin polarization map in Fig. 4(c) is in good agreement with the experimental one [see Fig. 4(b)] and reveals a negative polarization at the top sites and a positive polarization at the fcc/hcp sites for this energy. It is important to 

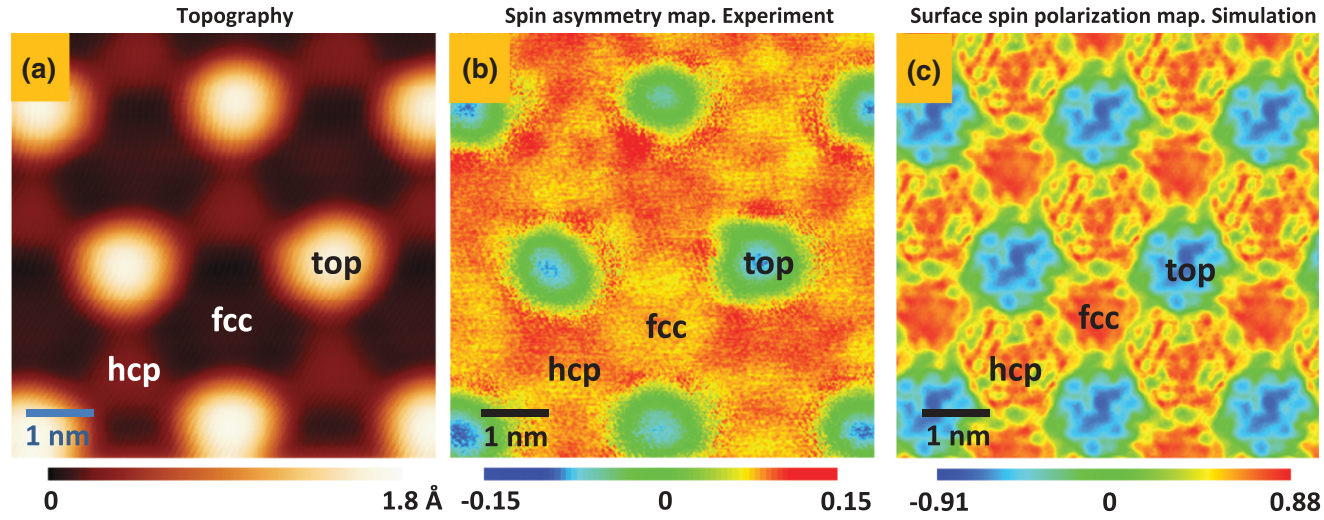

FIG. 4. (Color) Local effective spin polarization of the graphene layer. (a) Topography of an intercalation region (5 nA, $-1.0 \mathrm{~V})$. (b) Spin asymmetry map (see text) of the region shown in (a) which reveals the inversion of the effective local spin polarization between the top sites and fcc/hcp sites $(5 \mathrm{nA},-1.0 \mathrm{~V})$. (c) Simulated surface spin polarization map (Ref. 36). Panels (b) and (c) define a magnetic Moiré pattern of the graphene-Co/Ir(111) intercalation region for an applied bias voltage of $-1.0 \mathrm{~V}$.

note that this specific spin contrast between the top and fcc/hcp sites arises only from the decay of the $\pi$-type electronic states in the narrow energy interval $(25 \mathrm{meV})$ used to simulate the spin-resolved $d I / d V$ maps.

Our DFT calculations show that due to a strong graphenecobalt interaction at the fcc and hcp sites, a graphene unit cell acquires a total magnetic moment of $-1.36 \mu_{B}$ (spin-down electrons) leading to an antiferromagnetic coupling to the Co layer beneath. ${ }^{38,39}$ At top sites, where the graphenecobalt interaction is significantly weaker, the graphene has a much smaller total magnetic moment of $+0.14 \mu_{B}$ (spin-up electrons) and couples ferromagnetically to the Co layer. Note that the magnetic Moiré pattern defined by the variations of magnetic moments between the fcc, hcp, and top sites is apparently inverted with respect to the asymmetry maps shown in Fig. 4, i.e., a negative value at the hcp/fcc sites and a positive at the top sites. This difference arises due to the energydependent differences in the decay length of the $\pi$ orbitals in the different spin channels. In other words, while the total magnetic moments of graphene ${ }^{39}$ are obtained by summing over all occupied states up to the Fermi energy, the simulated spin polarization maps represent the decay in vacuum of the magnetization density corresponding to an energy interval used to record and simulate these maps. Interestingly, the orientation of the total magnetic moments of the graphene with respect to cobalt islands follows the general rule of an antiferromagnetic coupling for near magnetic moments at the fcc/hcp sites and a ferromagnetic coupling for distant magnetic moments at the top sites.

In summary, by combining cutting-edge SP-STM experiments and state-of-the-art first-principles calculations, we present a detailed characterization of the atomic structure and magnetic properties of the cobalt-intercalated graphene/Ir(111) system. The graphene layer presents a magnetic Moiré pattern with a high corrugation on the underlying cobalt monolayer. The graphene/Co/Ir(111) heterostructures exhibit an out-of-plane magnetization direction (easy axis) with an extremely high magnetic anisotropy energy. Such a high coercive field combined with the inert nature of the graphene-terminated surfaces makes the hybrid graphene/Co/Ir(111) system an ideal hard magnet for a new class of TMR or GMR technology. Furthermore, our approach opens a new route towards the observation of the magnetic properties of graphene. Here, the graphene is incorporated in a well-defined geometry and has a complex magnetic structure, which leads to a variation of the local spin polarization on the atomic scale. Such a surface may in the future be exploited to energy-selectively inject spin currents with different spin signs by choosing appropriate adsorption sites for spin-active adsorbates such as single atoms, magnetic molecules, or metal clusters.

\section{ACKNOWLEDGMENTS}

We gratefully acknowledge the Hamburg Cluster of Excellence NANOSPINTRONICS, the ERC Advanced Grant FURORE, the DFG via SFB668, the SPP1243, and Grant No. WI 1277/25. The computations have been performed on the high-performance computers JUROPA and JUGENE operated by the Jülich Supercomputing Centre at the Forschungzentrum Jülich.

\footnotetext{
*jbrede@physnet.uni-hamburg.de

†n.atodiresei@fz-juelich.de

${ }^{1}$ K. S. Novoselov, A. K. Geim, S. V. Morozov, D. Jiang, Y. Zhang, S. V. Dubonos, I. V. Grigorieva, and A. A. Firsov, Science 306, 666 (2004).
}

${ }^{2}$ J. Martin, N. Akerman, G. Ulbricht, T. Lohmann, J. H. Smet, K. von Klitzing, and A. Yacoby, Nat. Phys. 4, 144 (2008).

${ }^{3}$ Y. Zhang, V. W. Brar, Ç. O. Girit, A. Zettl, and M. F. Crommie, Nat. Phys. 5, 722 (2009). 
${ }^{4}$ V. W. Brar, R. Decker, H.-M. Solowan, Y. Wang, L. Maserati, K. T. Chan, H. Lee, Ç. O. Girit, A. Zettl, S. G. Louie, M. L. Cohen, and M. F. Crommie, Nat. Phys. 7, 43 (2011).

${ }^{5}$ H. Zabel and S. A. Sólin, Graphite Intercalation Compounds, Springer Series in Material Sciences, Vol. 14 (Springer-Verlag, New York, 1990).

${ }^{6}$ N. B. Hanney, T. H. Geballe, B. T. Matthias, K. Andres, P. Schmidt, and D. MacNair, Phys. Rev. Lett. 14, 225 (1965).

${ }^{7}$ T. E. Weller, M. Ellerby, S. S. Saxena, R. P. Smith, and N. T. Skipper, Nat. Phys. 1, 39 (2005).

${ }^{8}$ G. C. Csànyi, P. B. Littlewood, A. H. Nevidomskyy, C. J. Pickard, and B. D. Simons, Nat. Phys. 1, 42 (2005).

${ }^{9}$ A. M. Shikin, D. Farias, and K.-H. Rieder, Europhys. Lett. 44, 44 (1998).

${ }^{10}$ A. M. Shikin, D. Farias, V. K. Adamchuck, and K.-H. Rieder, Surf. Sci. 424, 155 (1999).

${ }^{11}$ L. Meng, R. Wu, H. Zhou, G. Li, Y. Zhang, L. Li, Y. Wang, and H.-J. Gao, Appl. Phys. Lett. 100, 083101 (2012).

${ }^{12}$ C. Riedl, C. Coletti, T. Iwasaki, A. A. Zakharov, and U. Starke, Phys. Rev. Lett. 103, 246804 (2009).

${ }^{13}$ L. Huang, Y. Pan, L. Pan, M. Gao, W. Xu, Y. Que, H. Zhou, Y. Wang, S. Du, and H.-J. Gao, Appl. Phys. Lett. 99, 163107 (2011).

${ }^{14}$ C. Enderlein, Y. S. Kim, A. Bostwick, E. Rotenberg, and K. N. Horn, New J. Phys. 12, 033014 (2010).

${ }^{15}$ Z.-H. Pan, J. Camacho, M. H. Upton, A. V. Fedorov, C. A. Howard, M. Ellerby, and T. Valla, Phys. Rev. Lett. 106, 187002 (2011)

${ }^{16}$ G. Profeta, M. Calandra, and F. Mauri, Nat. Phys. 8, 131 (2012).

${ }^{17}$ O. V. Yazyev, Rep. Prog. Phys. 73, 056501 (2010).

${ }^{18}$ N. Levy, S. A. Burke, K. L. Meaker, M. Panlasigui, A. Zettl, F. Guinea, A. H. Castro Neto, and M. F. Crommie, Science 329, 544 (2010).

${ }^{19}$ L. V. Dzemiantsova, M. Karolak, F. Lofink, A. Kubetzka, B. Sachs, K. von Bergmann, S. Hankemeier, T. O. Wehling, R. Frömter, H. P. Oepen, A. I. Lichtenstein, and R. Wiesendanger, Phys. Rev. B 84, 205431 (2011).

${ }^{20}$ M. Jullière, Phys. Lett. A 54, 225 (1975).

${ }^{21}$ P. Grünberg, R. Schreiber, Y. Pang, M. B. Brodsky, and H. Sowers, Phys. Rev. Lett. 57, 2442 (1986).
${ }^{22}$ M.N. Baibich, J. M. Broto, A. Fert, F. Nguyen Van Dau, F. Petroff, P. Etienne, G. Creuzet, A. Friederich, and J. Chazelas, Phys. Rev. Lett. 61, 2472 (1988).

${ }^{23}$ R. Wiesendanger, Rev. Mod. Phys. 81, 1495 (2009).

${ }^{24}$ S. Grimme, J. Comput. Chem. 27, 1787 (2006).

${ }^{25}$ N. Atodiresei, V. Caciuc, P. Lazic, and S. Blügel, Phys. Rev. Lett. 102, 136809 (2009).

${ }^{26}$ J. E. Bickel, F. Meier, J. Brede, A. Kubetzka, K. von Bergmann, and R. Wiesendanger, Phys. Rev. B 84, 054454 (2011).

${ }^{27}$ A. T. N'Diaye, S. Bleikamp, P. J. Feibelman, and T. Michely, Phys. Rev. Lett. 97, 215501 (2006).

${ }^{28}$ M. Batzill, Surf. Sci. Rep. 67, 83 (2012).

${ }^{29}$ S. Marchini, S. Günther, and J. Wintterlin, Phys. Rev. B 76, 075429 (2007).

${ }^{30}$ C. Busse, P. Lazić, R. Djemour, J. Coraux, T. Gerber, N. Atodiresei, V Caciuc, R. Brako, A. T. N'Diaye, S. Blügel, J. Zegenhagen, and T. Michely, Phys. Rev. Lett. 107, 036101 (2011).

${ }^{31}$ A. B. Preobrajenski, M. L. Ng, A. S. Vinogradov, and N. Mårtensson, Phys. Rev. B 78, 073401 (2008).

${ }^{32}$ M. Gyamfi, T. Eelbo, M. Wasniowska, and R. Wiesendanger, Phys. Rev. B 85, 205434 (2012).

${ }^{33}$ B. Borca, S. Barja, M. Garnica, M. Minniti, A. Politano, J. M. Rodriguez-Garcia, J. J. Hinarejos, D. Farias, A. L. Vazquez de Parga, and R. Miranda, New J. Phys. 12, 093018 (2010).

${ }^{34}$ C. Xia, S. Watcharinyanon, A. A. Zakharov, R. Yakimova, L. Hultman, L. I. Johansson, and C. Virojanadara, Phys. Rev. B 85, 045418 (2012).

${ }^{35}$ D. Wortmann, S. Heinze, P. Kurz, G. Bihlmayer, and S. Blügel, Phys. Rev. Lett. 86, 4132 (2001).

${ }^{36}$ See Supplemental Material at http://link.aps.org/supplemental/ 10.1103/PhysRevB.87.041403 for maps.

${ }^{37}$ J. Brede, N. Atodiresei, S. Kuck, P. Lazic, V. Caciuc, Y. Morikawa, G. Hoffmann, S. Blügel, and R. Wiesendanger, Phys. Rev. Lett. 105, 047204 (2010).

${ }^{38}$ N. Atodiresei, V. Caciuc, P. Lazic, and S. Blügel, Phys. Rev. B 84, 172402 (2011)

${ }^{39}$ The calculated total magnetic moments of the fcc/hcp and top sites have been obtained by integrating the total spin-polarized charge density in the atomic spheres of the hard PAW pseudopotentials and summing over all carbon atoms in these regions. 